\section{Titanium Foams Fabricated for Bone-Tissue Engineering Applications}

Titanium and its alloys are widely used as biological implants because of their superior biocompatibility, corrosion resistance, and lower modulus. However, the elastic moduli of these materials are still high when compared to those of human bone, particularly cancellous (porous) bone. Metallic foams are an option since the porous structure can more closely resemble that of bone. The optimum pore structure for these foams has been determined to be $\sim 200-500 \mu \mathrm{m}$ for permeation, attachment, and growth of osteoblasts. Producing metallic foams with pore sizes in this range using methods such as investment casting has proven difficult. Now, C.E. Wen and coworkers from the National Institute of Advanced Industrial Science and Technology, Japan, have fabricated titanium foams by using a powder metallurgical process incorporating space-holder particles to form a porous structure. They reported their work in the October issue of the Journal of Materials Research.

Pure titanium powder (purity $\geq 99.9 \%$ ) was used along with ammonium hydrogen carbonate powder as the space-holder material. The size of the ammonium hydrogen carbonate powder was selected to be 200-500 $\mu \mathrm{m}$, with the size of the titanium powder selected to be $<45 \mu \mathrm{m}$. The two were mixed, compacted using a pressure of $100 \mathrm{MPa}$, and then heat-treated and sintered to burn out the ammonium hydrogen carbonate. This resulted in the formation of titanium foams with relative densities ranging from 0.20 to 0.65 . Scanning electron microscopy (SEM) revealed a bimodal porous structure with macropores in the 200-500- $\mathrm{mm}$ range and micropores in the several micron-size range.

Compression tests showed that the mechanical properties of the titanium foams with relative densities of $0.20-0.30$ (Young's modulus 2.90-3.40 GPa; compressive strength 25-53 $\mathrm{MPa}$ ) were close to those of cancellous bone. The femoral head, for example, has a Young's modulus of $2.9 \mathrm{GPa}$ and a compressive strength of $68 \mathrm{MPa}$. The compressive properties of the foams with relative densities of 0.50-0.65 were similar to those of cortical (surface) bone. The results indicate that the mechanical properties of the titanium foam can be tailored by selecting the appropriate density.

Biocompatibility of the foams was tested by using an alkaline treatment on the samples first to yield a bioactive titania layer on exposed surfaces. The samples were then immersed in simulated body fluid that has ion concentrations similar to those of human plasma for in vitro assessment. SEM observations after six days of immersion indicated that a bonelike apatite layer formed throughout the foam at the cell edge surfaces of the pores. This indicated excellent permeability of the open-cell structure of the foam thereby endowing bioactivity throughout the foam.

The researchers said that their titanium foams appear to be promising biological implant materials for human bone-tissue engineering by virtue of their excellent biomechanical properties and bioactivity.

GOPAL RAO

\section{Covalent Nanoassemblies of Carbon Nanotubes and DNA Oligo-Nucleotides Synthesized}

The ability of single stranded (ss) DNA to selectively bind to a complementary strand combined with its ready availability make it an ideal material for "bottom up" nanoassembly construction. The stability of such assemblies is enhanced when the individual components are joined by covalent bonds. Recently, professor Robert Hamers, graduate student Sarah Baker, and co-workers in the Department of Chemistry at the University of Wisconsin-Madison, have demonstrated a covalent adduct of ssDNA and single-walled carbon nanotubes (SWNTs). The researchers observed that the covalent interactions were stable even at temperatures that would normally remove noncovalently bound DNA, and that covalent linking of DNA to the nanotubes also increased the solubility of the adduct in aqueous buffer solution. More importantly, the ssDNA-SWNT adducts could be very selectively and reversibly hybridized to other ssDNA molecules in solution, showing good biochemical accessibility. According to Hamers, "[T]hese nanostructures have a number of potential uses, such as nano building blocks in complex nanostructures, or in highly sensitive, reversible biosensors."

As reported in Nano Letters (Web release October 5, 2002), the first step in the synthesis of the covalent adduct was to functionalize the surface of the nanotubes with carboxylic acid groups. The acid groups were then converted to amide functionalities which were treated with the cross-linker succinimidyl 4(N-maleimidomethyl)cyclohexane-1carboxylate (SMCC) to produce maleimide functionalized nanotubes. In the last step, the maleimide groups were covalently attached to ssDNA. The assemblies were detected by labeling the ssDNA with a fluorescein dye group and measuring its photoluminescence (PL), or by linking unlabeled ssDNA to the tubes and then hybridizing the ssDNA-SWNT adducts with dye labeled DNA. In either case, the observed PL comes only from dye labeled DNA that is either directly bonded to the nanotubes or is hybridized to the ssDNA-SWNT adducts, not from the solution itself.

Hybridization experiments using complementary ssDNA and 4-base mismatched ssDNA showed that the covalently bound DNA maintains its specificity of binding, and that the DNA hybridization remains reversible. The specificity of hybridization proves that the DNA strand is available for hybridization, and is not intercalated inside or wrapped around the outside of the nanotube. This result demonstrates that despite being strongly bound to a SWNT, the DNA retains all the chemical properties of a free molecule. Thus, the covalent attachment would still allow the utilization of the ssDNA-SWNT adduct in very selective binding assays or the construction of complex nanostructures, and would result in improved stability of such systems.

GREG KHITROV

\section{Large, Crack-Free Photonic Films Developed}

Periodic dielectric superstructures are attracting interest, particularly for the formation of photonic bandgaps. However, application is limited because of the difficulty of preparing large, monocrystalline photonic structures with low defect density. Researchers from the Department of Chemistry at the University of Mainz have demonstrated a method for the preparation of large, crack-free photonic films. As reported in the October issue of Chemistry of Materials, B. Griesebock, M. Egen, and R. Zentel have developed a method to crystallize poly(methyl methacrylate) (PMMA) colloids on molten metals ( $\mathrm{Ga}$ or $\mathrm{Hg}$ ) as liquid substrates, which resulted in crack-free, nearly monocrystalline (low defect density) photonic-crystal films of millimeter size.

The researchers performed the crystallization by spreading several drops of a colloidal suspension onto the surface of the liquid substrate, then drying the film for $24 \mathrm{~h}$ at room temperature. The absence of cracks was checked by optical and scanning electron microscopy. With this method, the researchers prepared crack-free crystals of $\sim 1 \mathrm{~cm}^{2}$ area on liquid gallium and liquid mercury substrates. Polymer colloids prepared from poly(ethyl methacrylate), poly tert-butyl methacrylate, or fluorinated methacrylates also produced the same results. 